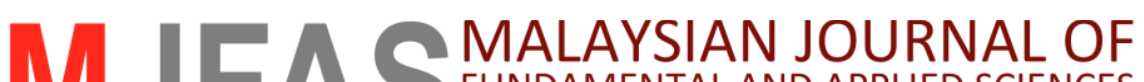 FUNDAMENTAL AND APPLIED SCIENCES

\section{Heat and mass transfer of steady magnetohydrodynamics mixed convection of dusty fluid flow with chemical reaction past an exponentially stretching sheet}

\author{
Siti Nur Haseela Izani ${ }^{\mathrm{a},},{ }^{,}$, Anati Ali ${ }^{\mathrm{b}}$ \\ a Department of Mathematical Sciences, Faculty of Science, Universiti Teknologi Malaysia, 81310 UTM Johor Bahru, Johor, Malaysia \\ b Department of Mathematical Sciences, Faculty of Science, Universiti Teknologi Malaysia, 81310 UTM Johor Bahru, Johor, Malaysia \\ * Corresponding author: snhaseela_izani@yahoo.com
}

\section{Article history}

Received 18 February 2017

Accepted 2 April 2017

Graphical abstract

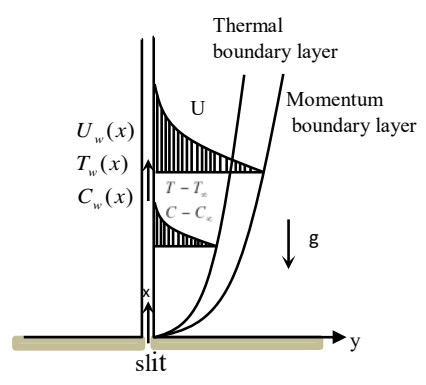

\begin{abstract}
An analysis has been carried out to study a problem of the chemical reaction effects on magnetohydrodynamics (MHD) mixed convective boundary layer flow with a fluid-particle suspension due to an exponentially stretching sheet. The effects of magnetic field and mass transfer are taken into account for the first time in the dusty fluid over the exponentially stretching sheet. The governing partial nonlinear differential equations corresponding to the momentum, energy and concentration are converted into a system of ordinary differential equations by using similarity transformations. The relevant dimensionless equations are then solved numerically using Runge-Kutta-Fehlberg fourth fifth order method (RKF45) with the help of Maple symbolic software. The influence of physical parameters on the velocity, temperature and concentration distributions for both phases were discussed numerically and presented in details through plotted graphs and tables. Also, the numerical values of skin friction coefficient, Nusselt and Sherwood number of the governing parameters are analyzed and discussed in details. The outcomes show that the reaction parameter affects the fluid flow whereas the magnetic field retards the fluid flow. A comparative study of the present results with the previous study provides an excellent agreement.
\end{abstract}

Keywords: Dusty fluid, heat and mass transfer, exponentially stretching sheet, chemical reaction, fluid-particle suspension

(C) 2017 Penerbit UTM Press. All rights reserved

\section{INTRODUCTION}

The study on free, forced and mixed convection under the influence of different physical conditions past a continuous stretching surface has gained much attention by many researchers due to its numerous industrial applications. Few examples of such industrial manufacturing processes are glass-fibre/paper production, the extraction of plastic sheet, plastic films drawing, hot/cold rolling and the metal spinning. Wide varieties of this problem are dealing with heat and mass transfer involving boundary layer concept as reported in the literature. The study found that during some processes, the stretching motion and the simultaneous of heating and cooling have crucial effects on the quality of the final products. The boundary layer is normally defined as a thin layer of fluid near the neighborhood of the solid boundary when a real fluid flows past a body.

The idea of boundary layer concept over a moving continuous solid surface (sheet issuing) was very well documented by Sakiadis [1, 2], who derived the approximate and exact solutions for the laminar velocity field. Motivated by Sakiadis's work, analytical and experimental approach of the heat transfer characteristics effects on a continuous moving surface has been analysed by Tsou [3]. Works on finding the solution to linear stretching sheet problem have also been done by Crane [4].
However, there were also investigations on the problem of boundary layer flow that use a non-standard stretching. A paper by Gupta and Gupta [5] on the heat and mass transfer on stretching sheet surface may be referred. The authors pointed out that this geometry may not always be linear. This assuming has been agreed by Kumaran and Ramanaiah [6] in the short article entitled 'A Note on the Flow over a Stretching Sheet'. In this paper, they considered quadratic stretching sheet for solving the boundary layer flow problem. Magyari and Keller [7] presented a similarity solution using both analytical and numerical for the heat and mass transfer of a flow over an exponentially stretching sheet. Sajid and Hayat [8] solved analytically the problem of viscous fluid in boundary layer flow and heat transfer by considering radiation effect. The research of viscous fluid flow over an exponentially stretching sheet has been extended by Ishak [9] and Bidin and Nazar [10].

Later on, many researchers have made an attempt to study the effect of buoyancy force and chemical reaction on boundary layer flow such as by Pal [11], Ali and Yousef [12], Sharada and Shankar [13], Srinivasacharya and Ramreddy [14], Patil et al. [15] and Bhattacharyya [16]. The analytical and numerical results were obtained and it is shown that both buoyancy force and chemical reaction affects the fluid flow. It is worth mentioning that in most of the chemical engineering 
process, a chemical reaction is present in between a foreign mass and the fluid. Hence, the study of heat and mass transfer in the presence of chemical reaction effect is also significant in chemical and hydrometallurgical.

The previous works focus on the effects of magnetic field parameter, mixed convection parameter and also chemical reaction parameter that affects the single-phase fluid flow characteristic. In facts, interest in the two-phase boundary layer flow has increased considerably in recent years due to the circumstance of these fluids in many industrial and technological applications. This scenario happened because single phase mechanisms are not relevant to be used to describe the nature of fluids that exist in daily life. Theoretically, the analysis of fluid flows embedded with dust particles is more interesting due to the assumptions made in the component form.

The simplest model for two-phase fluids flow was formerly proposed by Saffman [17] that discussed the stability of laminar flow of a dusty gas. In that research, the author proposed the equation that describes the flow of the gas-particle mixture. The author is confident that the proposed model and assumption on dust particles may offer some interest to the researchers worldwide. This is true when there are many researchers studied the multiphase flow in the different form of geometries and considered some effects that act on the flow motion. For instance, extensive studies on the topic of two-phase flow specifically related with Saffman's model have been conducted by Nayfeh [18], Marble [19], Chakrabati [20], Soo [21] and Drew [22].

Also, without considering any effects on the flow, Siddiqa et al. [23] investigated the mathematical formulation of two-phase natural convection flow of dusty fluid in details. The study refers to a wide range of Prandtl number $(0.005-1,000)$ and they found that when mass concentration parameter or Prandtl number is high, a very thin boundary layer is created. In addition, many researchers interested in the investigation of multiphase flow of fluid embedded with dust particles by considering different physical conditions include Venkataraman et al. [24], Krishna et al. [25], Isa and Ali [26] and Gireesha et al. [27,28].

However, all above literature survey limited their interest to the study of heat transfer boundary layer flow. The analysis of heat and mass transfer of fluid-particle suspensions is significant for a variety of reasons related to many practical applications. Saidu et al. [29] studied the effects of magnetohydrodynamics on the convective flow of dusty viscous fluid in the presence of volume fraction. Very recently, K.L Krupa et al. [30] numerically analyzed the heat and mass transfer of dusty fluid flows past a linearly stretching sheet. The authors, however, do not consider buoyancy force and chemical reaction in their investigation.

To date, the problem on mixed convection flow of a dusty fluid with chemical reaction effect past over exponentially stretching sheet has not been addressed. The governing partial nonlinear different equations of the flow, heat and mass transfer are converted into a system of ordinary differential equations by using similarity transformation. The transformed equations are then solved by using a numerical method. The effects of several dimensionless governing parameters on flow, temperature and concentration profiles are presented. We summarized our results in the following tables and then conclude our findings.

\section{FORMULATION OF THE PROBLEM}

\section{Assumptions}

This present work is considered a steady two-phase fluid flow by Saffman's model. The fluid is a two-dimensional incompressible flow past an exponentially stretching sheet. Along with this, heat and mass transfer are assumed to take place in the flow. The motion of fluid phase is influenced by buoyancy force. Also, some assumptions have been made for particle suspension [19]. The particles are uniformly distributed throughout the fluid. The shape of dust particles is a sphere.
The particles are rigid and all having the same mass and radius. However, the particles are considered as non-interacting and we neglect the volume fraction of solid particles in this work. Not to mention that the number density of the dust particle is constant throughout the flow. In view of the Cartesian coordinate system, $\mathrm{x}$-axis is taken in the direction along the stretching sheet while $y$-axis is perpendicular to it. The plate is assumed to be stretched with exponential velocity $U_{w}(x)=U_{o} \mathrm{e}^{x / L}$, having a surface at maintained temperature $T_{w}(x)=T_{\infty}+\left(T_{o}-T_{\infty}\right) \mathrm{e}^{x / 2 L}$ and exponential concentration distribution, $C_{w}(x)=C_{\infty}+\left(C_{o}-C_{\infty}\right) \mathrm{e}^{x / 2 L}$ where $U_{0}, T_{o}, C_{o}$ are constant and $\mathrm{L}$ is the reference length. $T_{\infty}$ and $C_{\infty}$ are the temperature and concentration far away from the stretching surface, respectively.

\section{Governing Equations}

Under this assumption, the flow of a dusty fluid are governed by the following equations

For the fluid phase:

$$
\frac{\partial u}{\partial x}+\frac{\partial v}{\partial y}=0
$$

$$
\begin{aligned}
u \frac{\partial u}{\partial x}+v \frac{\partial v}{\partial y}= & v \frac{\partial^{2} u}{\partial y^{2}}+\underbrace{\frac{K N}{\rho}\left(u_{p}-u\right)}_{\text {extra force }}-\frac{\sigma B^{2} u}{\rho} \\
& +\underbrace{g \beta^{*}\left(T-T_{\infty}\right)}_{\text {mixed convection }}+g \beta\left(C-C_{\infty}\right)
\end{aligned}
$$

For the dust phase:

$$
\begin{aligned}
& \frac{\partial u_{p}}{\partial x}+\frac{\partial v_{p}}{\partial y}=0 \\
& u_{p} \frac{\partial u_{p}}{\partial x}+v_{p} \frac{\partial u_{p}}{\partial y}=\frac{K}{m}\left(u-u_{p}\right)
\end{aligned}
$$

together with boundary condition on velocity,

$$
\begin{array}{ll}
u=U_{w}(x) \quad v=0, & \text { at } y=0, \\
u \rightarrow 0, \quad u_{p} \rightarrow 0, \quad v_{p} \rightarrow v & \text { as } y \rightarrow \infty .
\end{array}
$$

Where $(u, v)$ and $\left(u_{p}, v_{p}\right)$ are the velocity components of the fluid and particle phase along $x$ and $y$, respectively. $v=\mu / \rho$ is the kinematic viscosity of the fluid, $C$ and $T$ are the concentration and temperature of the fluid inside the boundary layer. $N, \rho, \sigma, B, g$ and $\beta^{*}$ are the number density of the dust particles, fluid density, electrical conductivity, magnetic field strength, acceleration of gravity and coefficient of thermal expansion. $K=6 \pi \mu a$ denotes the Stokes's drag which is a frictional force of viscosity on small spherical particles moving through a viscous fluid at a lower rate of motion. Notice that in equation (1), there exist an extra force term due to the interaction between fluid and particle.

The following similarity variable $\eta$ and non-dimension variables are introduced in order to convert the governing partial differential equations to a set of coupled non-linear ordinary differential equations (ODE) 
$u=U_{o} e^{x / L} f^{\prime}(\eta), u_{p}=U_{o} e^{x / L} F^{\prime}(\eta)$,

$v=-\sqrt{\frac{U_{o} v}{2 L}} e^{x / 2 L}\left(f(\eta)+\eta f^{\prime}(\eta)\right), \eta=\sqrt{\frac{U_{o}}{2 v L}} e^{x / 2 L} y$,

$v_{p}=-\sqrt{\frac{U_{o} v}{2 L}} e^{x / 2 L}\left(F(\eta)+\eta F^{\prime}(\eta)\right), B=B_{0} e^{x / 2 L}$.

Here, $f(\eta)$ is a non-dimensional stream function. Using the similarity equations above, Equation 1 and 3 are satisfied. Equation 2 and 4 are then reduced to the following non-linear ODE of fluid motion

$$
\begin{aligned}
& f^{\prime \prime \prime}(\eta)+f(\eta) f^{\prime \prime}(\eta)-2 f^{\prime}(\eta)^{2}+2 \ell \beta_{v}\left(F^{\prime}(\eta)-f^{\prime}(\eta)\right) \\
& -M f^{\prime}(\eta)+2 \lambda(\theta(\eta)+H \phi(\eta))=0 \\
& F(\eta) F^{\prime \prime}(\eta)-2 F^{\prime}(\eta)^{2}+2 \beta_{v}\left(f^{\prime}(\eta)-F^{\prime}(\eta)\right)=0
\end{aligned}
$$

(8)

with the new boundary conditions in terms of $f(\eta)$

$$
\begin{aligned}
& f(\eta)=0, \quad f^{\prime}(\eta)=1 \quad \text { at } \eta=0, \\
& f^{\prime}(\eta)=0, F^{\prime}(\eta)=0, F(\eta)=f(\eta)+\eta f^{\prime}(\eta)-\eta F^{\prime}(\eta) \text { as } \eta \rightarrow \infty
\end{aligned}
$$

where $\ell=m N / \rho$ is the dust particles mass concentration parameter, $\beta_{v}=L / \tau_{v} U_{0}$ is the local fluid-particle interaction parameter for velocity, $M=2 \sigma B_{0}{ }^{2} L / \rho U_{0} \quad$ is the magnetic parameter, $H=\beta\left(C_{w}-C_{\infty}\right) / \beta^{*}\left(T_{w}-T_{\infty}\right)$ is the buoyancy ratio parameter, $\lambda=R i=G r / \operatorname{Re}^{2}$ is the mixed convection parameter in which $G r=g \beta^{*}\left(T_{w}-T_{\infty}\right) L^{3} / v^{2}$ is the thermal Grashof number and $\operatorname{Re}=U_{w} L / v$ is the local Reynolds number. Noting that, when $\lambda<0$, the problem involve opposing flow and when $\lambda>0$, the problem involve assisting flow.

\section{Heat and Mass Transfer Analysis}

The governing steady, heat and mass transfer equations for both phases in the presence of chemical reaction are given by

For the fluid phase:

$u \frac{\partial T}{\partial x}+v \frac{\partial T}{\partial y}=\frac{\kappa}{\rho c_{p}} \frac{\partial^{2} T}{\partial y^{2}}+\frac{\rho_{p} c_{m}}{\rho c_{p} \tau_{T}}\left(T_{p}-T\right)+\frac{\rho_{p}}{\rho c_{p} \tau_{v}}\left(u_{p}-u\right)^{2}$

$u \frac{\partial C}{\partial x}+v \frac{\partial C}{\partial y}=D_{m} \frac{\partial^{2} C}{\partial y^{2}}+\frac{\rho_{p}}{\rho \tau_{c}}\left(C_{p}-C\right)-K r *\left(C-C_{\infty}\right)$

For the dust phase:

$\rho_{p} c_{m}\left(u_{p} \frac{\partial T_{p}}{\partial x}+v_{p} \frac{\partial T_{p}}{\partial y}\right)=-\frac{\rho_{p} c_{m}}{\tau_{T}}\left(T_{p}-T\right)$

$u_{p} \frac{\partial C_{p}}{\partial x}+v_{p} \frac{\partial C_{p}}{\partial y}=\frac{1}{\tau_{c}}\left(C-C_{p}\right)$

where $\tau_{v}=m / K, \kappa, D_{m}, K r^{*}$ correspond to the relaxation time of dust phase, thermal conductivity, diffusion coefficient, chemical reaction coefficient. $\tau_{T}$ and $\tau_{c}$ are the thermal equilibrium time (time needed by a dust particle to move its temperature and concentration slightly in order to achieve the desired fit into the fluid). $c_{p}$ and $c_{m}$ are the specific heat of fluid and dust particles, $C_{p}$ and $T_{p}$ are the concentration and temperature of the dust particle inside the boundary layer, respectively. $T_{\infty}$ and $C_{\infty}$ are the temperature and species concentration of the fluid far away from the wall respectively. $\rho_{p} / \rho \tau_{c}\left(C_{p}-C\right)$ represent the mass diffusion between the fluid and dust particles.

The boundary conditions of temperature and concentration are given by:

$T=T_{\infty}+\left(T_{o}-T_{\infty}\right) e^{x / 2 L} \theta(\eta), T_{p}=\left(T_{o}-T_{\infty}\right) e^{x / 2 L} \theta_{p}(\eta)+T_{\infty}$

$C=C_{\infty}+\left(C_{o}-C_{\infty}\right) e^{x / 2 L} \theta(\eta), C_{p}=\left(C_{o}-C_{\infty}\right) e^{x / 2 L} \theta_{p}(\eta)+C_{\infty}$,

$T=T_{w}(x), \quad C=C_{w}(x), \quad$ at $y=0$,

$T \rightarrow T_{\infty}, \quad T_{p} \rightarrow T_{\infty} \quad C \rightarrow C_{\infty}, \quad C_{p} \rightarrow C_{\infty}$ as $y \rightarrow \infty$.

Setting $\theta(\eta), \theta_{p}(\eta), \phi(\eta), \phi_{p}(\eta)$ as the dimensionless temperature and concentration for fluid and dust variable;

$\begin{aligned} \theta(\eta) & =\frac{T-T_{\infty}}{T_{w}-T_{\infty}}, & \theta_{p}(\eta) & =\frac{T_{p}-T_{\infty}}{T_{w}-T_{\infty}} \\ \phi(\eta) & =\frac{C-C_{\infty}}{C_{w}-C_{\infty}}, & \phi_{p}(\eta) & =\frac{C_{p}-C_{\infty}}{C_{w}-C_{\infty}},\end{aligned}$

Inserting equation (15) into equation (10) - (14), yield the transformed ODE;

$\theta^{\prime \prime}(\eta)+\operatorname{Pr}\left(f(\eta) \theta^{\prime}(\eta)-f^{\prime}(\eta) \theta(\eta)\right)+2 \operatorname{Pr} \beta_{T} \ell \gamma$

$\left(\theta_{p}(\eta)-\theta(\eta)\right)+2 \operatorname{Pr} \beta_{v} E c \ell\left(F^{\prime}(\eta)-f^{\prime}(\eta)\right)^{2}=0$

$\phi^{\prime \prime}(\eta)+S c\left(f \phi^{\prime}-f^{\prime} \phi-2 K r \phi+2 l \beta_{c}\left(\phi_{p}-\phi\right)=0\right.$

$F^{\prime}(\eta) \theta_{p}(\eta)-F(\eta) \theta_{p}^{\prime}(\eta)+2 \beta_{T}\left(\theta_{p}(\eta)-\theta(\eta)\right)=0$

$F^{\prime} \phi_{p}-F \phi_{p}^{\prime}+2 \beta_{c}\left(\phi_{p}-\phi\right)=0$

with boundary conditions for the temperature and concentration

$\begin{array}{ll}\theta(\eta)=1 \quad & \phi(\eta)=1 \quad \text { at } \quad \eta=0, \\ \theta(\eta) \rightarrow 0, & \theta_{p}(\eta) \quad \phi(\eta) \rightarrow 0, \quad \phi_{p}(\eta) \rightarrow 0 \quad \text { as } \quad \eta \rightarrow \infty\end{array}$

where $\operatorname{Pr}=\mu C_{p} / k$ is the Prandtl number, $\beta_{T}=L / \tau_{T} U_{0}$ and $\beta_{c}=L / \tau_{c} U_{0}$ are the local fluid-particle interaction parameter for heat transfer and concentration, $E c=U_{0}{ }^{2} e^{2 x / L} / c_{p}\left(T_{w}-T_{\infty}\right)$ is the Eckert number, $S c=v / D_{m}$ is the Schmidt number, $K r=L K r^{*} / U_{0}$ is the chemical reaction parameter. A prime denotes differentiation with respect to $\eta$.

\section{NUMERICAL SOLUTION OF THE PROBLEM}

The non-linear partial differential equations (PDE) are converted into a set of non-linear ODE (7)-(8), (16)-(19) by using suitable similarity transformation. In order to generate the numerical solution, the study used a RKF45 method. The appropriate boundary layer thickness of $\eta \rightarrow \infty$ was chosen prudently.

We compare the values of the dimensionless temperature gradient $-\theta^{\prime}(0)$ with Magyari and Keller [7], Srinivasacharya and Ramreddy [14], 
Pal [11] and Chaudhary et al. [31] in order to verify the accuracy of the employed method as indicate in Table 1.

Table 1 Values of $-\theta^{\prime}(0)$ for several values of $\operatorname{Pr}$ when $\beta_{v}=\beta_{t}=\beta_{c}=M=H=\lambda=E c=S c=K r=0$.

\begin{tabular}{|c|c|c|c|c|c|}
\hline $\mathrm{Pr}$ & $\begin{array}{c}\text { Magyari and Keller } \\
\text { [7] }\end{array}$ & $\begin{array}{c}\text { Srinivasacharya and Ramreddy } \\
{[14]}\end{array}$ & $\begin{array}{l}\text { Pal } \\
{[11]}\end{array}$ & $\begin{array}{c}\text { Chaudhary et.al } \\
{[31]}\end{array}$ & Present result \\
\hline 1 & 0.95478 & 0.95478 & 0.95478 & 0.9559 & 0.95481 \\
\hline 5 & 2.50014 & 2.50015 & 2.50013 & & 2.50013 \\
\hline 8 & 3.24212 & 3.24218 & 3.24212 & & 3.24212 \\
\hline 10 & 3.66037 & 3.66043 & 3.66037 & & 3.66037 \\
\hline
\end{tabular}

Table 2 Numerical values of $f^{\prime \prime}(0),-\theta^{\prime}(0)$ and $-\phi^{\prime}(0)$ for different values of $\operatorname{Pr}, E c, l, M, S c, \lambda, \beta_{c}, \beta_{v}, \beta_{T}$ and $K r$.

\begin{tabular}{|c|c|c|c|c|c|c|c|c|c|c|c|}
\hline $\operatorname{Pr}$ & $E c$ & $M$ & $S c$ & $\lambda$ & $\beta_{c}$ & $\beta_{v}$ & $\beta_{T}$ & $K r$ & $f^{\prime \prime}(0)$ & $-\theta^{\prime}(0)$ & $-\phi^{\prime}(0)$ \\
\hline 0.72 & & & & & & & & & -0.34796 & 0.90004 & 1.53966 \\
\hline 1.0 & & & & & & & & & -0.39532 & 1.07782 & 1.53134 \\
\hline 3.0 & & & & & & & & & -0.56261 & 1.95763 & 1.50540 \\
\hline 5.0 & & & & & & & & & -0.63616 & 2.56980 & 1.49627 \\
\hline & 0 & & & & & & & & -0.68608 & 3.12171 & 1.49090 \\
\hline & 0.2 & & & & & & & & -0.68508 & 3.11109 & 1.49102 \\
\hline & 0.3 & & & & & & & & -0.68457 & 3.10577 & 1.49107 \\
\hline & 0.4 & & & & & & & & -0.68407 & 3.10045 & 1.49113 \\
\hline & & 0 & & & & & & & -0.30911 & 3.13836 & 1.52855 \\
\hline & & 1.0 & & & & & & & -0.68104 & 3.06850 & 1.49148 \\
\hline & & 2.0 & & & & & & & -0.99551 & 3.00682 & 1.46241 \\
\hline & & 3.0 & & & & & & & -1.27151 & 2.95106 & 1.43863 \\
\hline & & & 0 & & & & & & -0.22653 & 3.17575 & 0.09999 \\
\hline & & & 0.5 & & & & & & -0.57793 & 3.09594 & 1.03644 \\
\hline & & & 0.8 & & & & & & -0.64720 & 3.07763 & 1.32644 \\
\hline & & & 1.5 & & & & & & -0.74281 & 3.05169 & 1.84561 \\
\hline & & & & 0 & & & & & -1.64589 & 2.88757 & 1.41675 \\
\hline & & & & 1.0 & & & & & -0.68104 & 3.06850 & 1.49148 \\
\hline & & & & 2.0 & & & & & 0.18982 & 3.19387 & 1.54662 \\
\hline & & & & 3.0 & & & & & 1.00633 & 3.29255 & 1.59202 \\
\hline & & & & & 0 & & & & -0.66772 & 3.06954 & 1.47110 \\
\hline & & & & & 1.0 & & & & -0.68259 & 3.06808 & 1.49957 \\
\hline & & & & & 1.5 & & & & -0.68343 & 3.06785 & 1.50386 \\
\hline & & & & & 2.0 & & & & -0.68395 & 3.06771 & 1.50651 \\
\hline & & & & & & 0 & & & -0.66712 & 3.12629 & 1.49327 \\
\hline & & & & & & 1.0 & & & -0.68952 & 3.06245 & 1.49043 \\
\hline & & & & & & 1.5 & & & -0.69526 & 3.06341 & 1.48976 \\
\hline & & & & & & 2.0 & & & -0.69943 & 3.06599 & 1.48929 \\
\hline & & & & & & & 0 & & -0.68050 & 3.06001 & 1.49153 \\
\hline & & & & & & & 1.0 & & -0.68136 & 3.07331 & 1.49145 \\
\hline & & & & & & & 1.5 & & -0.68156 & 3.07633 & 1.49143 \\
\hline & & & & & & & 2.0 & & -0.68171 & 3.07838 & 1.49141 \\
\hline & & & & & & & & 0.2 & -0.63486 & 3.08088 & 1.26159 \\
\hline & & & & & & & & 0.3 & -0.65212 & 3.07627 & 1.34311 \\
\hline & & & & & & & & 0.5 & -0.68104 & 3.06850 & 1.49148 \\
\hline & & & & & & & & 1.0 & -0.73351 & 3.05433 & 1.80597 \\
\hline
\end{tabular}

\section{RESULTS AND DISCUSSION}

A study on two-dimensional hydromagnetic fluid flow and mass transfer of dusty fluid over an exponentially stretching sheet in presence of chemical reaction is considered. Throughout the numerical study, we used $\quad B_{v}=B_{t}=B_{c}=0.5, E c=1, \ell=0.1, \lambda=1, K r=0.5, P r=7, S c=1$ and $M=1$. The main point of this section is to put on view the behavior of physical parameters, namely fluid-particle parameter for velocity, temperature, concentration ( $\left.B_{v}, B_{t}, B_{c}\right)$, Eckert number ( $\left.E c\right)$, dust particle mass concentration parameter $(\ell)$, mixed convection parameter $(\lambda)$, chemical reaction parameter ( $K r)$, Prandtl number $(P r)$, Schmidt number $(S c)$ and magnetic number $(M)$ on the flow, temperature and concentration profiles. The values of the friction factor, Nusselt and Sherwood number are also given in tabular form.

Figures 1a-1c shows the effect of fluid-particle interaction parameters $B_{v}$ , $B_{t}, B_{c}$ on velocity, temperature and concentration profile for fluid and dust phase. We observed that the fluid- particles interaction significantly increases the velocity, temperature and concentration profile for dust phase. Meanwhile, velocity, temperature and concentration profile gradually decrease with an increase $B_{v}, B_{t}, B_{c}$ for fluid phase. Physically, this may happen due to large values of $B_{v}, B_{t}, B_{c}$ which causes the relaxation time, $\tau$ takes some time to adjust to change in fluid velocity. Then, the velocity for both phases will be the same. Figure 2 is plotted for both fluid and dust phase to see the variation of Eckert number on the 
temperature profile. This figure shows that a rise in the value of $E c$ parameter increases the temperature profile for both phases. In fact, enhancement in Eckert number parameter implies more heat energy dissipating throughout the boundary layer due to the frictional heating (drag force). Hence, this will cause the temperature field increase for both fluid and dust phase.

The influences of mass concentration of dust particle on velocity and concentration distributions are shown in Figures 3a-3b. We observed that the effects of dust particles mass concentration are to decelerate the velocity and concentration of the fluid flow at any point for both phases. This may happen due to the internal friction effects occurs in between the particle and clean air.

To illustrate the effects of mixed convection parameter that act on the fluid, the variation of velocity, temperature and concentration profiles are demonstrated in Figures $4 \mathrm{a}-4 \mathrm{c}$, respectively.

We observed that the increase in mixed convection parameter accelerate the fluid flow for both fluid and dust phase but reduce the temperature and concentration profile. Also, in the matter of mixed convection, we have observed that temperature is low for assisting flow and high for opposing flow. In consequence, as the buoyancy effect increase, the cooling surface will increase, hence reduce the temperature profile.

Figure $5 \mathrm{a}$ and $5 \mathrm{~b}$ show the chemical reaction effect on velocity and concentration profiles. Both figures illustrate that the velocity and concentration decrease with an increase in the values of $K r$. This behavior may happen due to an enhancement in the interfacial mass transfer, which then diminishes the species concentration. Thus, it is true to conclude that chemical reaction affects the diffusion rate.

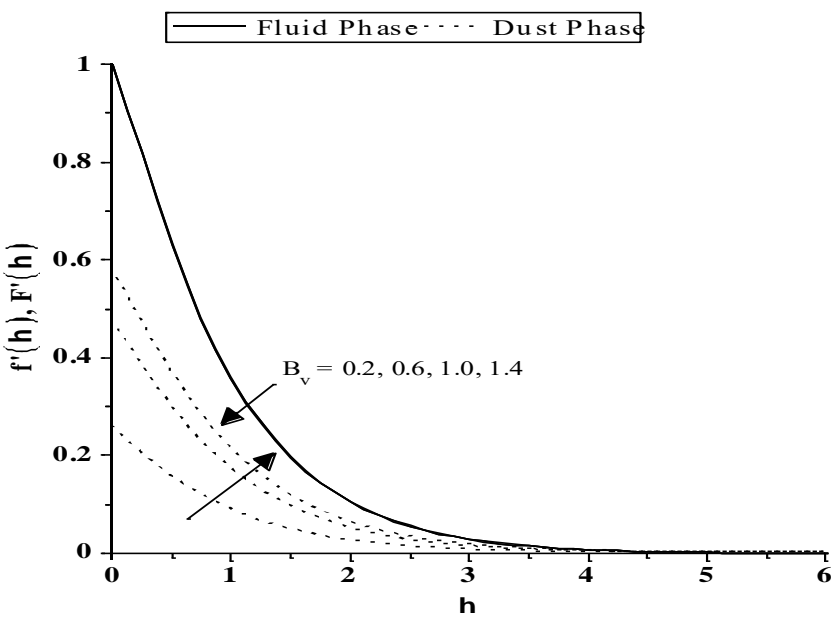

Fig. 1a Effect of $B_{v}$ on velocity profile against $\eta$

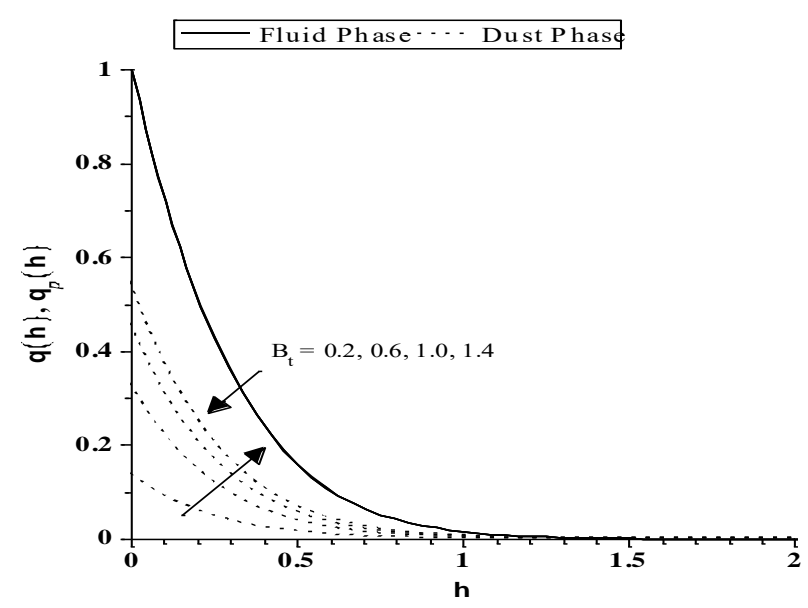

Fig. 1b Effect of $B_{t}$ on temperature profile against $\eta$

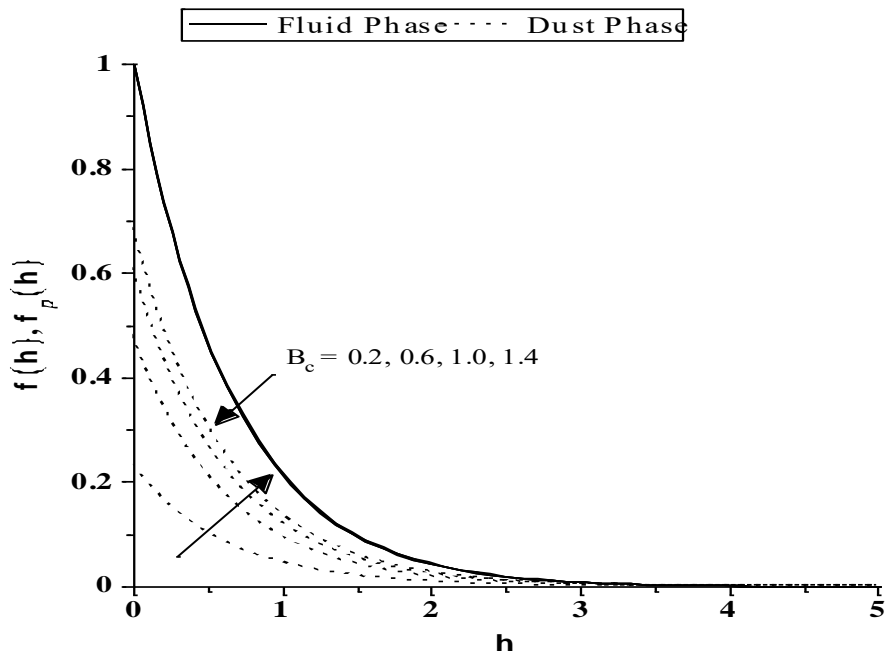

Fig. 1c Effect of $B_{c}$ on concentration profile against $\eta$

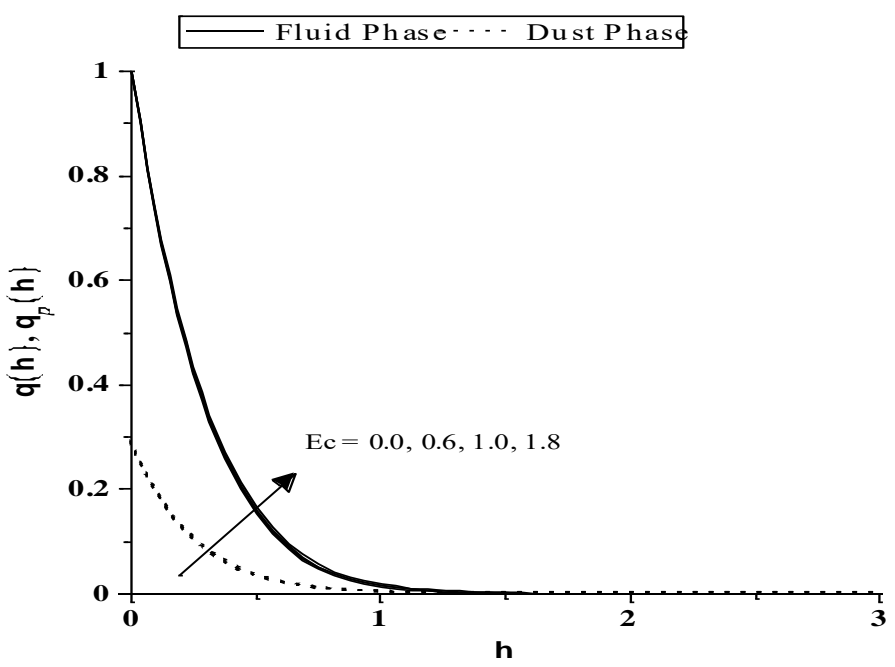

Fig. 2 Effect of $E c$ on temperature profile against $\eta$

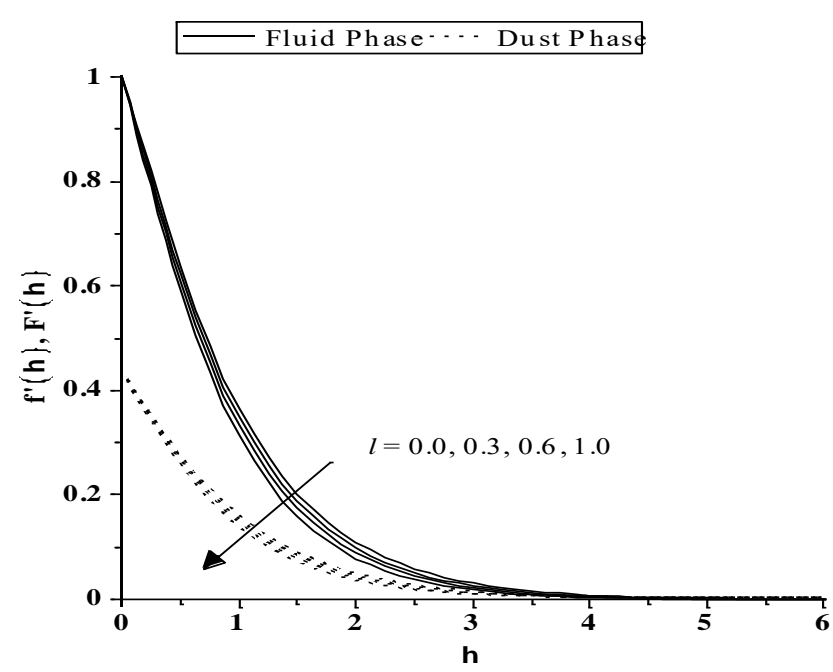

Fig. 3a Effect of $\ell$ on velocity profile against $\eta$ 


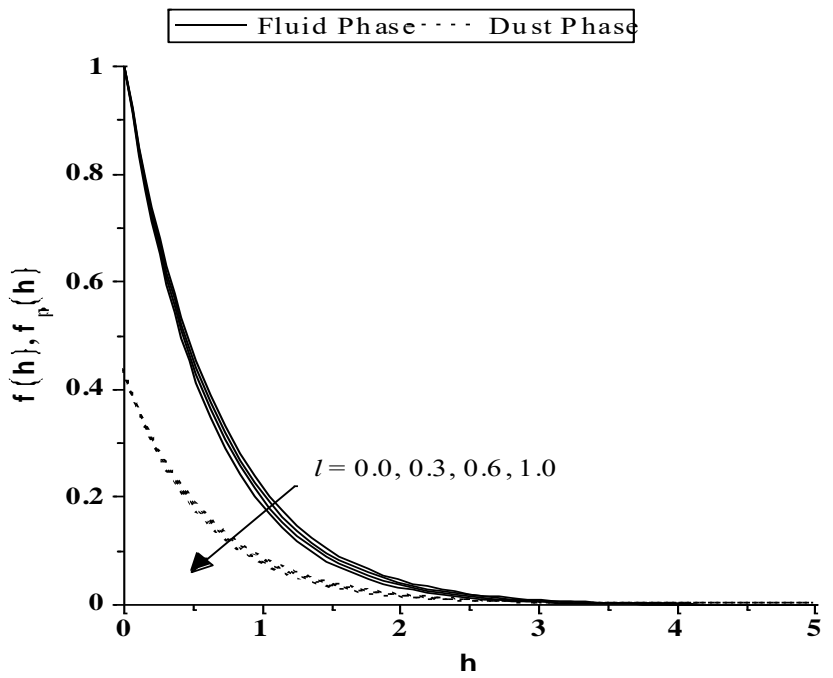

Fig. $3 \mathbf{b}$ Effect of $\ell$ on concentration profile against

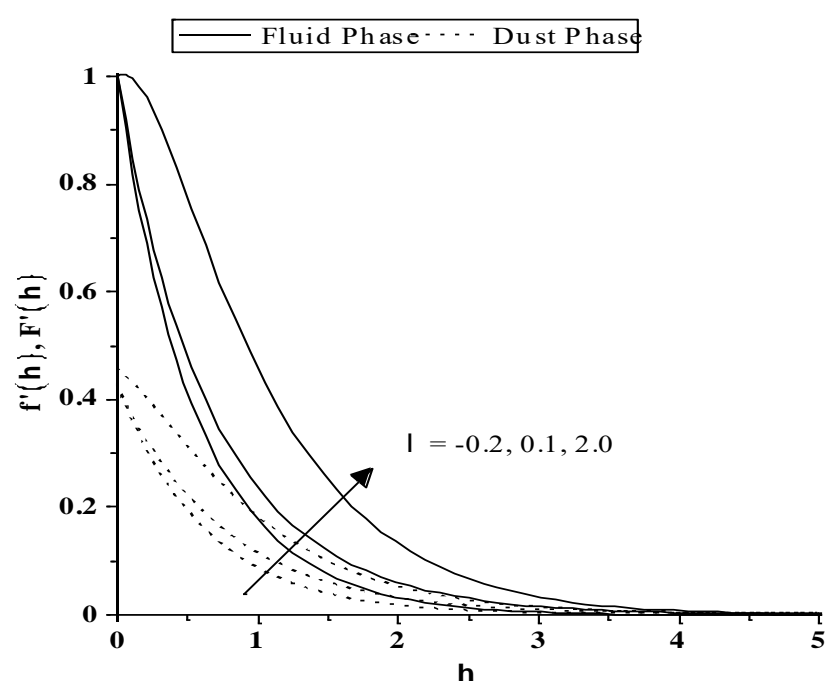

Fig. 4a Effect of $\lambda$ on velocity profile against $\eta$

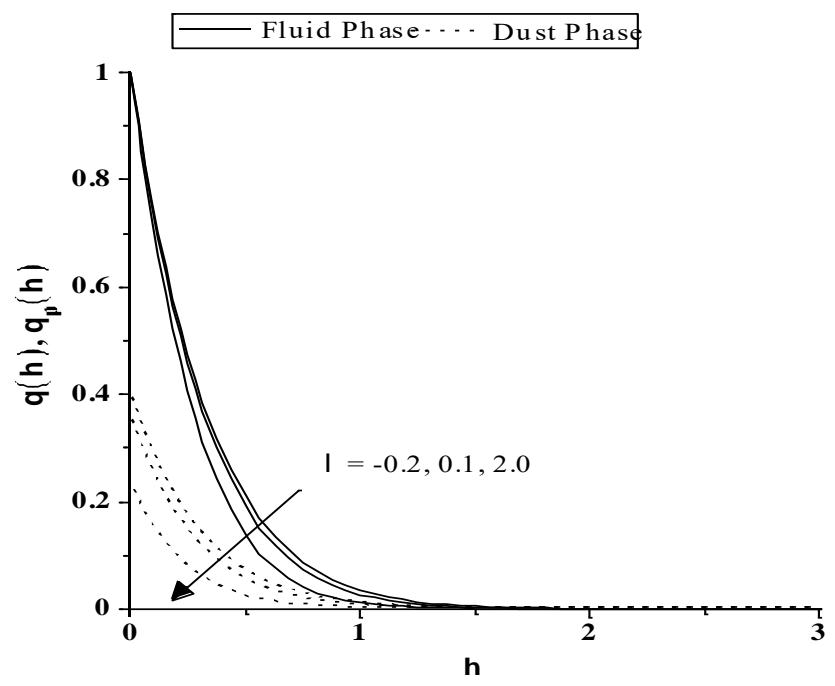

Fig. $4 \mathrm{~b}$ Effect of $\lambda$ on temperature profile against $\eta$

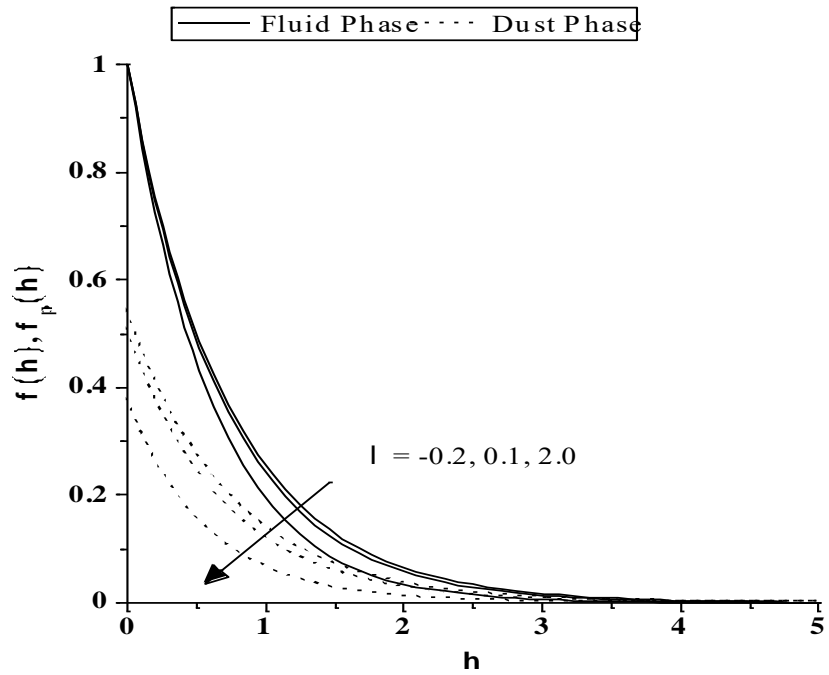

Fig. 4c Effect of $\lambda$ on concentration profile against $\eta$

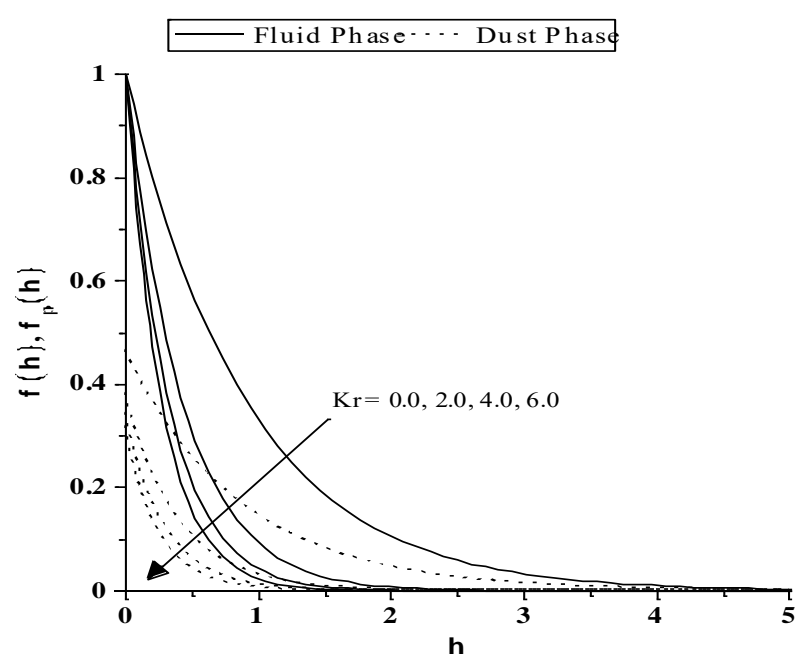

Fig. 5a Effect of $K r$ on concentration profile against $\eta$

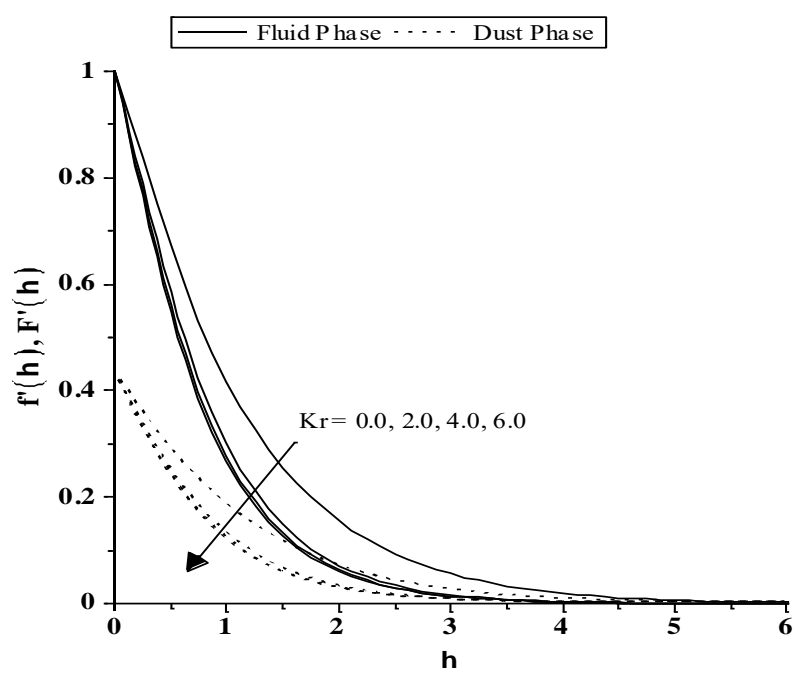

Fig. 5b Effect of $K r$ on velocity profile against $\eta$ 


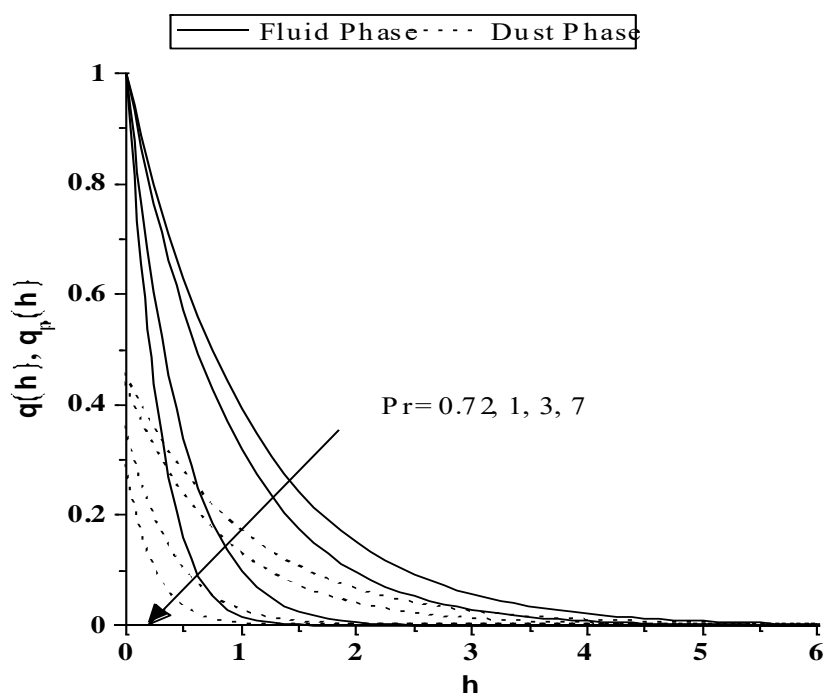

Fig. 6 Effect of Pr on temperature profile against $\eta$

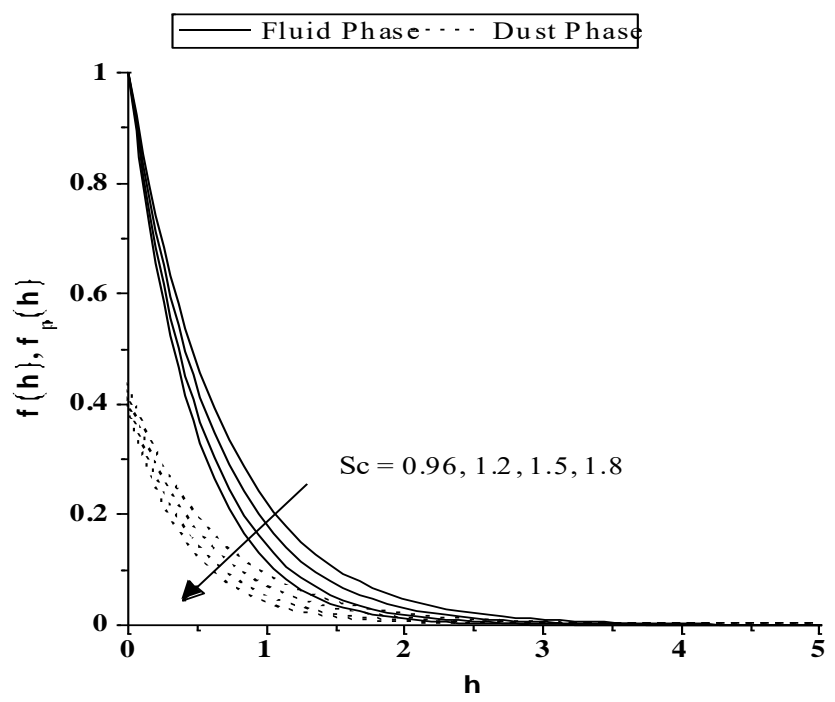

Fig. 7 Effect of $S c$ on concentration profile against $\eta$

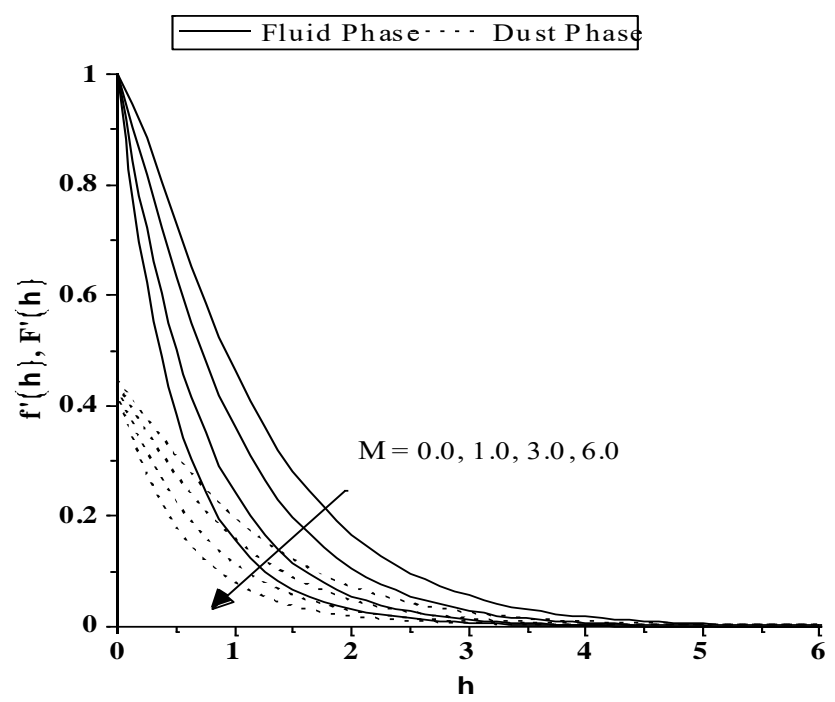

Fig. 8a Effect of $M$ on velocity profile against $\eta$

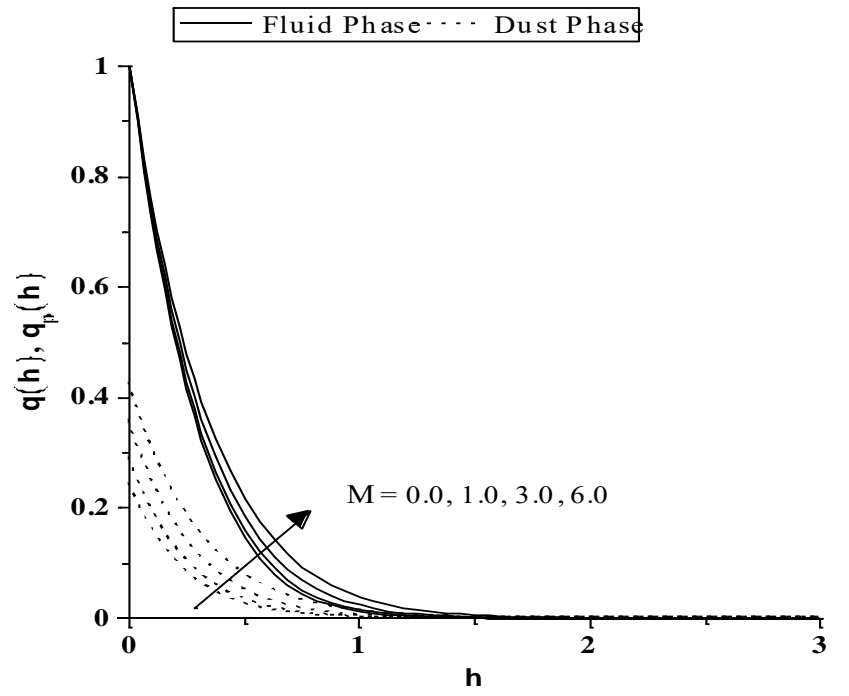

Fig. $\mathbf{8 b}$ Effect of $M$ on temperature profile against $\eta$

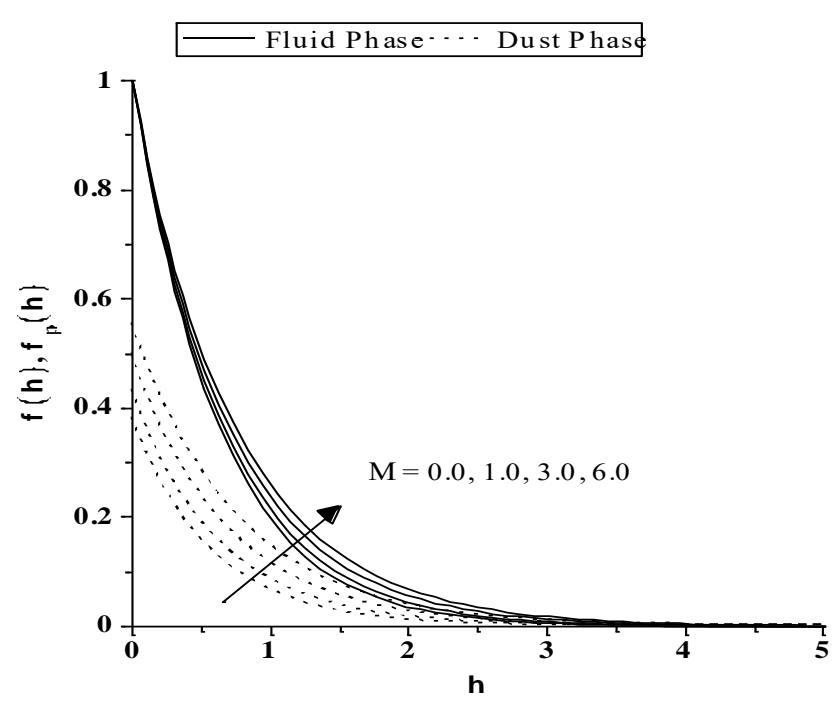

Fig. 8c Effect of $M$ on concentration profile against $\eta$

Figure 6 and 7 depict the effect of Prandtl number and Schmidt number on temperature and concentration profile for both phases respectively. Increasing the values of $\operatorname{Pr}$ number decrease the temperature profile. In heat transfer problem, we found that smaller Prandtl number leads to lower the viscosity and higher the thermal conductivity. This is true due to the physical fact; Prandtl number is the ratio of momentum diffusivity to thermal diffusivity. As a result, the ability of thermal diffusivity is dropped when $\operatorname{Pr}$ enhances in values correspond to the thinner thermal boundary layer for both phases. Thus, it is worth to conclude that value of $\operatorname{Pr}$ depends on the thermal conductivity of the particular fluid.

Besides, the concentration profile in the boundary layer is declined as expected by increasing the values of Schmidt number. In view of mass transfer problem, one should know that the mass analog of the Prandtl number is Schmidt number, which is a ratio of viscosity to mass diffusivity. Physically, the mass diffusivity declines when $S c$ increases. Due to that, the lower the Schmidt number, the higher the concentration of the species. 
The influence of magnetic field parameter on velocity, temperature and concentration profiles are shown in Figure 8. It shows that velocity profiles for both phases decrease with a rise in the values of $M$. This result is obvious because a resistive type force (so-called Lorentz force) will be produced by applying the transverse magnetic field normal to the flow direction in an electrically conducting fluid. Lorentz force has a tendency to delay the motion of fluid flow and boost the temperature field. As a result, the velocity profiles for both phases decelerates rapidly.

Table 2 depicts the variation of local skin friction coefficient $f^{\prime \prime}(0)$ local Nusselt number $-\theta^{\prime}(0)$ and Sherwood number $-\phi^{\prime}(0)$ for various values of $\operatorname{Pr}, E c, M, S c, \lambda, B_{v}, B_{t}, B_{c}$ and $K r$. A rise in the magnetic field parameter declines the skin friction drag, Nusselt number and Sherwood number. On the other hand, the skin friction drag, the variation of Nusselt number and Sherwood number increase with the increase of $\lambda$. Meanwhile, an increase in Eckert number parameter leads to decrease the values of the Nusselt number but enhances the skin friction coefficient and Sherwood number. The friction factor and Nusselt number decrease however, enhance the Sherwood number when the values of Schmidt number, local fluid-particle interaction for concentration and chemical reaction parameter increase. In addition, it is observed that the friction factor at the surface and mass transfer rate decreases with the increasing values of Prandtl number and local fluid-particle for velocity and temperature along with heat transfer rate.

\section{CONCLUSION}

Numerical solution corresponding to the phenomenon of heat and mass transfer of hydromagnetic dusty fluid flow with buoyancy force and reaction parameter over an exponentially stretching sheet has been discussed in this article. The behavior of physical parameters is shown in the form of velocity, temperature and concentration profile. The main results of the present analysis are concluded as follows:

1. The effect of mixed convection parameter, $\lambda$ beneficial for the fluid flow distribution. But it is unfavorable for temperature and concentration. We found that the negative values of $\lambda$ will slow down the flow motion.

2. The influences of the magnetic field, $M$ affects the flow behavior significantly.

3. A rise in the values of chemical reaction, retard the concentration profile. Hence, the reaction parameter and Schmidt affect the diffusion rate of heat and mass transfer problems.

4. The effects of dust particles mass concentration on fluid and dust phase reduce friction factor and increase the heat transfer rate. Thus, the characteristics of flow can be controlled greatly by altering $\ell$.

We believe that the theoretical study of fluid flow problems with fluidparticles suspension will give a fruitful knowledge to the real application worldwide and might be useful in controlling the flow, temperature and concentration distribution.

\section{ACKNOWLEDGEMENT}

The authors are very much thankful to the reviewers for their encouraging comments and suggestions to improve the present paper.

\section{REFERENCES}

[1] B. C. Sakiadis, AICHE J. 7 (1961) 26.

[2] B. C. Sakiadis, AICHE J. 7 (1961) 221

[3] F. K. Tsou, E. M. Sparrow, R. J. Goldstein, Int. J. Heat Mass Transf. 10 (1967) 219.

[4] L. J. Crane, Z. Angew, Math. Phys. 21 (1970) 645.

[5] P. S. Gupta, A. S. Gupta, Can. J. Chem. Eng. 55 (1977) 744.

[6] V. Kumaran, G. Ramanaiah, Acta Mech. 116 (1996) 229.

[7] E. Magyari, B. Keller, J. Phys. D: Appl. Phys. 32 (1999) 577.

[8] M. Sajid, T. Hayat, Int. Commun. Heat Mass 35 (2008) 347.

[9] A. Ishak, Sains Malays. 40 (2011) 391.

[10] B. Biliana, N. Roslinda, Eur. J. Sci. Res. 33 (2009) 710.

[11] D. Pal, Appl. Math. Comput. 217 (2010) 2356.

[12] M. Ali, F. Al-Yousef, Int. J. Heat Mass Transf. 45 (2002) 4241.

[13] K. Sharada, B. Shankar, World J. of Mechanics 5 (2015) 165.

[14] D. Srinivasacharya, C. RamReddy, J. Nonlinear Sci. 12 (2011) 60.

[15] P. M. Patil, S. Roy, E. Momoniat, Int. J. Heat Mass Transf. 100 (2016) 482.

[16] K. Bhattacharyya, J. Egyptian Math. Society 20 (2012) 223.

[17] P. G. Saffman, J. Fluid Mech. 13 (1962) 120.

[18] A. H. Nayfeh, AIAA J. 4 (1966) 1868.

[19] F. E. Marble, Annu. Rev. Fluid Mech. 2 (1970) 397.

[20] K. M. Chakrabarti, AIAA J. 12 (1974) 1136.

[21] S. L. Soo, Phys. Fluids 18 (1975) 263.

[22] D. A. Drew, Annu. Rev. Fluid Mech. 15 (1983) 261.

[23] S. Siddiqa, M. A. Hossain, S. C. Saha, Int. J. Numer. Method H 25 (2015) 1542.

[24] V. Venkataraman, K. Kannan, R. Dharmarajan, GJSFR 12 (2012).

[25] P. M. Krishna, V. Sugunamma, N. Sandeep, Commun. Appl. Sci. 1 (2013) 161.

[26] S. M. Isa, A. Ali, S. Shafie, AIP Conf. Proc. 1750 (2016).

[27] B. J. Gireesha, G. M. Pavithra, C. S. Bagewadi, Br. J. Math. Stat. Psychol. 2 (2012) 187.

[28] B. J. Gireesha, A. J. Chamkha, S. Manjunatha, C. S. Bagewadi, Int. J. Numer. Method H 23 (2013) 598.

[29] I. Saidu, M. M. Waziri, A. Roko, M. Hamisu, J. Eng. Appl. Sci. 5 (2010) 86.

[30] K. K. Lakshmi, B. J. Gireesha, R. S. Gorla, B. Mahanthesh, IJIM 8 (2016) 279.

[31] S. Chaudhary, S. Singh, S. Chaudhary, Appl. Math. 6 (2015) 295. 